\title{
L'ARGENTURE DE L'ANGUILLE : MÉTAMORPHOSE, ANTICIPATION, ADAPTATION.
}

\section{Y. A. FONTAINE}

Laboratoire de Physiologie Générale et Comparée, Muséum National d'Histoire Naturelle, URA 90 CNRS “Evolution des Régulations Endocriniennes», 7 rue Cuvier, 75231 Paris Cedex 05, France.

\section{RÉSUMÉ}

L'argenture est une métamorphose qui, au cours du cycle vital des anguilles, précède la migration marine de reproduction. Une meilleure connaissance de ce phénomène serait utile non seulement d'un point de vue fondamental en biologie du développement mais aussi d'un point de vue appliqué pour la gestion des populations de ce poisson.

On passe d'abord en revue un certain nombre de caractères très variés, qui ont été invoqués pour différencier les stades jaune et argenté, en s'attachant à la signification, pour le cycle vital, des nouveautés exprimées durant l'argenture ; la plupart de celles-ci apparaissent être des caractères adaptatifs qui anticipent sur les conditions de la migration marine.

On s'intéresse ensuite à la dynamique et au déterminisme neuroendocrinien de l'argenture. On suggère l'existence de plusieurs étapes qui se succéderaient du printemps à la fin de l'automne, de la préargenture à la descente des rivières (l'avalaison). En se référant aux rares données neuroendocrinologiques disponibles ainsi qu'à d'autres métamorphoses comme celles des amphibiens et la smoltification du saumon, on est conduit à proposer qu'un certain nombre d'hormones interviennent dans le déterminisme des étapes successives de l'argenture. Un des événements précoces pourrait être la stimulation de neurones hypothalamiques, avec sécrétion de neuropeptides hypophysiotropes. Une question particulièrement intéressante posée par l'argenture de l'anguille, et sur laquelle on insiste, est celle du couplage des déclenchements de la métamorphose et de la puberté.

Un scénario évolutif est proposé pour l'apparition du cycle vital si complexe des anguilles à partir de la vie entièrement marine qui devait être celle de leurs ancêtres comme elle l'est encore pour la plupart de leurs proches parents, les autres élopomorphes. $\mathrm{Ce}$ scénario implique en particulier l'acquisition de l'euryhalinité, puis "l'assimilation génétique" des processus d'abord mis en oeuvre lors de l'accommodation à l'eau de mer ; c'est dire que ces processus ont dû, au cours de l'évolution, être placés sous le contrôle de l'horloge du développement qui déclenche l'argenture; des, hypothèses neuroendocriniennes sont suggérées pour expliquer cette assimilation génétiqúe.

En conclusion, on s'interroge sur la définition morphologique et biochimique des étapes successives de l'argenture et sur la reconnaissance de ces étapes par des caractères, aisément détectables, plus précis et fiables que la coloration. Des progrès dans ces domaines permettraient de connaître par exemple, dès avant l'avalaison, la proportion du stock qui quittera les eaux douces dans l'année.

Mots-clés : anguille, métamorphose, migration, physiologie, évolution.

\section{EEL SILVERING : METAMORPHOSIS, ANTICIPATION, ADAPTATION.}

\section{SUMMARY}

Eel "silvering" is a metamorphosis which, during the life cycle of these fish, precedes the marine reproductive migration. A better knowledge of this phenomenon would be useful not only from a fundamental point of view in developmental biology but also from an applied point of view for the management of eel populations. 
First, we review a number of characters wich distinguish between yellow and silver stages with a special attention to the meaning, for the life cycle, of the novelties expressed during silvering; most of them appear to be adaptative characters which anticipate the conditions of the marine migration. Then, we take an interest in the dynamics and the neuroendocrine determination of silvering for which the existence of several successive steps is suggested, from presilvering to the catadromous freshwater journey ("avalaison" in French). The neuroendocrine data on silvering are rare so that one is led to use informations from other events such as amphibian metamorphoses and salmon smoltification. It appears likely that several hormones are involved in the determinism of silvering successive steps. One of the precocious events could be the stimulation of hypothalamic neurons with secretion of hypophysiotropic neuropeptides. An especially interesting point raised by eel silvering, the coupling between silvering and puberty triggerings, is discussed.

An evolutionary scenario is proposed for the appearance of the present complex eel life cycle from the purely marine life which was probably that of eel ancestors as it is still for most of their close relatives, the other elopomorphs. This scenario in particular involves the acquisition of euryhalinity and then the "genetic assimilation" of processus previously triggered during accomodation to sea water. That is to say that these processes should have been placed under the control of the developmental clock which initiates silvering; neuroendocrine hypotheses are suggested to explain this genetic assimilation.

In conclusion, one wonders about the morphological and biochemical definition of the successive steps of silvering and on the recognition of these steps by easily detectable characters, more precise and reliable than colouring. Progresses in these scopes would allow for instance to know before «avalaison» the proportion of a given stock which is likely to leave fresh waters in the year.

Key-words : eel, metamorphosis, migration, physiology, evolution.

\section{INTRODUCTION}

Après quelques cinq à quinze ans de vie sédentaire dans des eaux continentales ou littorales, et avant leur migration marine de reproduction, les anguilles passent du stade "jaune" au stade "argenté» (Figure 1). Cette "argenture" correspond à tout un ensemble de transformations morphologiques et physiologiques qui sépare, dans le cycle vital des anguilles (BERTIN, 1951 ; D'ANCONA, 1960 ; FONTAINE, 1985) deux étapes caractérisées par des modes de vie et des environnements très différents. II s'agit donc clairement d'une métamorphose (BERTIN, 1958 ; WALD, 1981 ; LECOMTE-FINIGER, 1990) et cette métamorphose présente nombre de caractères particulièrement intéressants. D'abord, c'est une seconde métamorphose par opposition à la métamorphose larvaire qui transforme le leptocéphale en civelle. Ensuite, c'est une métamorphose de rupture, de basculement de la croissance à la reproduction. Enfin, elle se prépare à l'aventure qu'est la longue migration en eau profonde (FONTAINE, 1989).

D'un point de vue fondamental, l'argenture de l'anguille constitue donc un modèle remarquable pour la biologie du développement. De plus, étant donné les différences profondes existant entre l'écophysiologie, les modes de capture, les qualités organoleptiques et les destins des anguilles jaunes et argentées, il semble important pour la gestion des stocks de pouvoir distinguer aisément et sûrement les deux stades. Enfin, une meilleure compréhension de l'argenture pourrait conduire à la maîtrise, qui serait d'un réel poids économique, du phénomène.

Malgré son intérêt, l'argenture de l'anguille est encore fort mal connue, mise à part la description d'un certain nombre de caractères qui différencient les stades jaune et argenté. Nous rappellerons d'abord les principales de ces différences et insisterons sur leur signification pour le cycle vital. Nous nous intéresserons ensuite à la dynamique et au déterminisme de l'argenture afin de formuler des hypothèses de travail, sur la base des rares données disponibles. Enfin nous proposerons un scénario pour l'acquisition de l'argenture au cours de l'Evolution. 


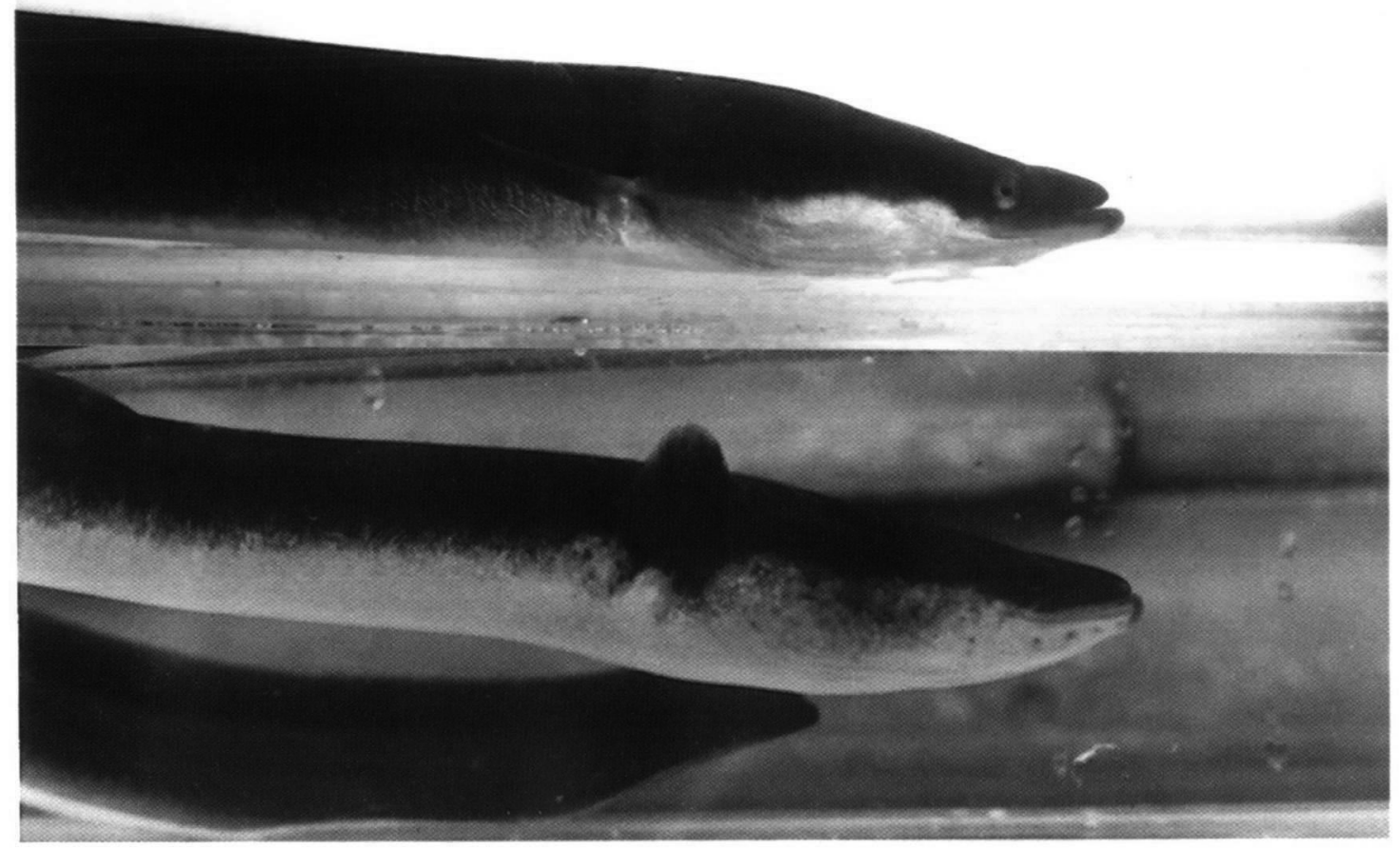

Figure 1 : Anguilles jaune et argentée.

Figure 1 : Yellow and silver eels.

Sauf indication contraire, nous considérerons seulement ici l'anguille européenne, Anguilla anguilla $L$. Les autres espèces, américaine (Anguilla rostrata Le Sueur) ou pacifiques, présentent des cycles vitaux similaires même si les longueurs des migrations sont très variables (TESCH, 1979).

\section{CARACTĖRES DISTINCTIFS DES STADES JAUNE ET ARGENTÉ}

\section{Peau et organes des sens}

II s'agit d'abord de la coloration du corps qui a donné leurs noms aux deux stades "jaune» et «argenté». En effet, durant la longue phase sédentaire de croissance, le dos est le plus souvent brun verdâtre et le ventre jaune tandis qu'ils sont respectivement brun noir et blanc argenté lorsque les poissons sont prêts à entreprendre leur migration marine de reproduction. Classiquement on admet que ces différences reflètent une augmentation de la mélanine dorsale et des purines ventrales (BERTIN, 1951). Pour PANKHURST et LYTHGOE (1982), le changement majeur est une redistribution des purines entre les couches externe et interne de chromatophores mais il reste possible qu'une augmentation de la mélanine intervienne à un stade final de l'argenture. En fait, les colorations de la peau peuvent largement varier, en particulier chez les "jaunes", si bien qu'elles ne peuvent constituer une base absolue pour la détermination des stades du développement (PANKHURST et LYTHGOE, 1982).

Selon BERTIN (1951, 1958), l'argenture s'accompagne d'un épaississement de la peau et d'un "accroissement général" des organes des sens. Quelques publications ont précisé ces évènements dans le cas de la ligne latérale (ZACCHEI et TAVOLARO, 1988), de l'épithélium olfactif (SORENSEN et PANKHURST, 1988, chez Anguilla 
rostrata) et surtout de l'œil. Afin de quantifier l'augmentation de la taille de ce dernier, PANKHURST (1982a) a défini un index oculaire (1O, en $\mathrm{mm}$ ) qui est calculé par la formule suivante : $[(A+B) / 4]^{2} \times[\pi / L] \times 10$ où $A$ et $B$ sont les diamètres horizontal et vertical (en $\mathrm{mm}$ ) tandis que $\mathrm{L}$ est la longueur de l'animal $(\mathrm{en} \mathrm{cm})$. Un 10 de 6,5 est la plus petite valeur trouvée par PANKHURST chez les anguilles «entreprenant leur migration". II convient de faire ici une parenthèse sur l'interprétation des travaux de cet auteur. En effet, celui-ci ne met pas directement en parallèle les différences qu'il étudie avec les colorations des animaux mais avec leur statut sexuel caractérisé par la taille des follicules ovariens. Quoi qu'il en soit, 10 est, grossièrement, environ 2 fois plus grand chez les individus qu'on peut raisonnablement supposer argentés que chez les jaunes supposés. En même temps que l'index oculaire, la surface de la rétine augmente ainsi que le nombre des bâtonnets (dont la densité ne change pas) tandis que la densité des cônes et des cellules nerveuses sous-jacentes diminue (PANKHURST et LYTHGOE, 1982). Les pigments rétiniens changent aussi. La substance dominante est respectivement la porphyropsine chez les jaunes et la rhodopsine chez les argentées ; la porphyropsine, association d'une opsine et du rétinal 2, présente une absorption maximale à $523 \mathrm{~nm}$ tandis que la rhodopsine, association d'une autre opsine avec le rétinal 1 , présente une absorption maximale à $482 \mathrm{~nm}$; ce changement peut être associé (BEATTY, 1975) ou non (WOOD et PARTRIDGE, 1993) à la différenciation de nouvelles cellules photoréceptrices.

\section{Vessie natatoire}

Les changements importants qui se produisent au niveau de la vessie natatoire ont été quantifiés chez Anguilla rostrata. Ils concernent la paroi de la vessie, dont la teneur en guanine augmente très significativement, d'environ $50 \%$ (KLECKNER, 1980), ainsi que les corps rouges. Ces derniers, organes pairs accolés à l'intérieur de la vessie natatoire et composés chacun d'une glande à gaz et d'un réseaú admirable, assurent la sécrétion de gaz à l'intérieur de la vessie natatoire. L'argenture correspond à un allongement $(2,5 \mathrm{x})$ des capillaires du réseau admirable et à une augmentation de leur section, sans changement de leur nombre (KLECKNER et KRUEGER, 1981).

\section{Métabolismes et comportement}

Les anguilles argentées jeûnent. II s'agit d'un jeûne physiologique observé aussi chez d'autres espèces de poissons à certaines étapes de leur cycle vital, en particulier chez le saumon (Salmo salar) lors de sa migration anadrome de reproduction, et il a été bien décrit par M. FONTAINE et OLIVEREAU (1962) : "Ce jeûne physiologique, lié à l'élaboration des produits génitaux, est fort différent du jeûne imposé et MISLIN l'a désigné sous le nom de jeûne synchonique, terme qui, issu du verbe grec "synchonomai", exprime l'idée d'écoulement et de refonte, c'est-à-dire cette condition très spéciale du saumon de montée qui réunit le catabolisme et l'utilisation de certaines substances à des fins énergétiques, mais aussi le transfert, la transformation et le réemploi de certains métabolites dans d'autres organes, gonades essentiellement." D'autres comportements distinguent l'anguille argentée de l'anguille jaune sédentaire. D'une part, il existe au cours de l'argenture une phase d'activité motrice qui peut conduire par exemple les poissons à sortir de l'eau et à cheminer dans l'herbe humide et qui pourrait correspondre à un état de sympathicotonie jouant un rôle dans le déclenchement de la migration (M. FONTAINE, 1975). D'autre part, il existe ultérieurement une phase d'asthénie qui résulte sans doute d'un déséquilibre osmotique (M. FONTAINE, 1975). En effet l'argenture induit des changements de l'osmorégulation. Il y a diminution du $\mathrm{Na}^{+}$et du $\mathrm{Cl}^{-}$dans le muscle et le sang, une déminéralisation due à la mise en route de mécanismes hypoosmorégulateurs alors que les poissons sont encore en eau douce (M. FONTAINE, 1975). Au niveau de la branchie, ces changements sont liés à l'augmentation du nombre des cellules à chlorure et de l'activité Na K-ATPasique (THOMPSON et SARGENT, 1978). De ces points de vue, l'anguille argentée est déjà un poisson d'eau de mer, milieu auquel elle s'accommode d'ailleurs bien plus vite et plus efficacement que l'anguille jaune (M. FONTAINE, 1975 ; THOMPSON et SARGENT, 1978). 
Des changements interviennent aussi en ce qui concerne le métabolisme intermédiaire (BOSTRÖM et JOHANSSON, 1972 ; LEWANDER et al., 1974 ; DAVE et al., 1974 ; BARNI et al., 1985 ; EGGINTON, 1986) avec, en particulier, une augmentation des lipides et des mitochondries dans les hépatocytes (BARNI et al., 1985) et dans les muscles (PANKHURST, $1982 \mathrm{~b}$; LARSSON et al., 1990). BOETIUS et BOETIUS (1985) ont montré que la part de l'énergie totale du corps due aux lipides est plus importante chez les argentées que chez les jaunes. L'ensemble de ces données a été récemment disćuté par LARSEN et DUFOUR (1993).

\section{Gonades}

L'argenture est marquée par une augmentation nette du poids des ovaires, le rapport gonadosomatique (RGS) passant de valeurs voisines de 0,3 à des valeurs voisines de 1,5 qui correspondent respectivement à des diamètres folliculaires d'environ 0,05 et $0,26 \mathrm{~mm}$ (FONTAINE et al., 1976 ; LOPEZ et FONTAINE, 1990). Pour PANKHURST (1982a), le diamètre folliculaire marquant la limite entre les animaux qu'il appelle respectivement “immatures" et "en cours de maturation sexuelle» est $0,10 \mathrm{~mm}$, une valeur correspondant à un index oculaire de 6,5 et, d'après notre expérience, à un RGS de 0,6 . Nous reviendrons plus loin sur l'évolution du RGS au cours de l'argenture. Nous avons précisé (FONTAINE et al ., 1976 ; LOPEZ et FONTAINE, 1990) les changements structuraux qui se produisent dans l'ovaire : augmentation du diamètre folliculaire, épaississement de l'enveloppe folliculaire, apparition de nombreuses vésicules lipidiques. La comparaison quantitative des données obtenues chez jaunes et argentées fait ressortir un phénomène assez spectaculaire. On s'aperçoit en effet que, durant l'argenture, le volume folliculaire est multiplié par 125 tandis que le RGS est multiplié seulement par 5,2 ; le nombre apparent de follicules pour une anguille de $100 \mathrm{~g}$ diminue donc de $96 \%$ (FONTAINE, données inédites). Ce calcul, effectué en supposant que tout l'ovaire est formé de follicules, n'est qu'approximatif puisque l'ovaire contient aussi des travées conjonctives et un tissu interstitiel, mais il montre qu'une atrésie folliculaire importante se produit durant l'argenture.

\section{Système endocrinien}

Un certain nombre de données suggèrent que les anguilles argentées présentent, par rapport aux anguilles jaunes, une hyperactivité des glandes interrénales et de la thyroïde (LELOUP-HATEY, 1964 ; FONTAINE, 1975 ; LELOUP et al., 1976). Nous reviendrons plus loin sur ces aspects de la métamorphose. Les ovaires d'anguilles jaunes et argentées diffèrent dans la réponse à un traitement gonadotrope par un extrait d'hypophyse de carpe : rapport gonadosomatique et diamètre folliculaire augmentent beaucoup moins vite chez les premières que chez les dernières (FONTAINE et al., 1976 ; LOPEZ et FONTAINE, 1990). De plus, après un tel traitement, des teneurs plasmatiques élevées d'œstradiol et de testostérone sont trouvées chez l'anguille argentée tandis que seul l'œstradiol est élevé chez la jaune (LELOUP-HATEYet al., 1989).

\section{Signification dans le cycle vital des caractères apparaissant avec l'argenture}

La stratégie vitale de l'anguille, acquise au cours de l'Evolution, est telle qu'elle doit concilier une croissance dans les eaux continentales (ou littorales) et une reproduction à quelque $6000 \mathrm{~km}$ de là, en Mer des Sargasses. De plus, deux types d'arguments indiquent que la migration marine doit se faire en profondeur; il s'agit d'abord de quelques rares observations ou captures (FONTAINE, 1989) ; il s'agit ensuite de résultats expérimentaux montrant que la profondeur (et elle seule parmi les facteurs de l'environnement étudiés) est capable de stimuler l'axe gonadotrope de l'anguille argentée (FONTAINE et al., 1985; DUFOUR, 1986 et ce numéro).

Dans ces conditions, nombre des caractères acquis au cours de l'argenture et que nous avons passés en revue apparaissent à l'évidence comme adaptatifs. La vie en eau de mer est facilitée par l'augmentation du nombre des cellules à chlorure branchiales et des capacités hypoosmorégulatrices. La vie en profondeur est facilitée à plusieurs égards. La capacité d'augmenter le contraste entre les colorations dorsale et ventrale permet un camouflage efficace. L'augmentation de la surface rétinienne et du nombre de bâtonnets, ainsi que les changements pigmentaires assurent une meilleure sensibilité à la faible lumière 
qui parvient en profondeur. Les changements au niveau de la vessie natatoire permettent à celle-ci de rester fonctionnelle sous haute pression : augmentation de la capacité du corps rouge à sécréter des gaz et diminution de la conductance de la paroi pour ceux-ci grâce au dépôt de guanine. Bien sûr, il convient, dans ce genre de situation, de se méfier d'une approche trop "panglossienne" de l'utilité des changements (GOULD et LEWONTIN, 1979). On peut pourtant suggérer aussi que des changements métaboliques, en particulier l'augmentation des réserves lipidiques, sont favorables au long voyage vers la Mer des Sargasses. Enfin, la diminution drastique du nombre des follicules ovariens peut augmenter la part des réserves consacrée à la migration en diminuant celle consacrée à la vitellogénèse.

Tous ces changements se produisent alors que les poissons sont encore dans leur phase de vie sédentaire, généralement dulcicole. Ils sont donc anticipateurs (M. FONTAINE, 1983). Les nouveaux caractères ne s'expriment pas en réponse à des changements de l'environnement. Au contraire, leur expression est sous la dépendance d'un programme de développement. Pour reprendre une terminologie définie par ailleurs (FONTAINE, 1994) il ne s'agit pas "d'accommodations" mais de l'expression "d'adaptats" - ou caractères adaptatifs - héréditaires. On peut enfin se demander si le rôle le plus fondamental de certains de ces adaptats anticipateurs n'est pas tant de préparer le voyage que de le déclencher. C'est ainsi que les changements osmorégulateurs qui préparent à la vie en eau de mer induisent, en eau douce, un déséquilibre, un conflit ; il en résulte une déminéralisation sans doute responsable de l'asthénie souvent observée et donc de l'aspect passif de la migration catadrome, de "l'avalaison" (M. FONTAINE, 1975). Plus généralement, et puisque la reproduction ne semble pouvoir avoir lieu qu'au terme d'une migration aux caractères bien précis, il est en effet essentiel pour la survie de l'espèce que des signaux imposent cette migration. Par exemple, une fois les poissons arrivés en eau de mer ou bien chez les individus qui se sont argentés dans les eaux marines littorales, d'autres signaux doivent déclencher la nage vers les eaux profondes. Les adaptats concernant la vessie natatoire ou les yeux pourraient jouer ce rôle en changeant le preferendum de lumière ou la flottabilité mais les mécanismes impliqués restent à déterminer.

\section{DYNAMIQUE ET DETERMINISME DE L'ARGENTURE}

Comme les autres métamorphoses (DICKHOFFet al., 1990), en particulier celles des amphibiens qui sont les mieux connues (WHITE et NICOLL, 1981), l'argenture de l'anguille est certainement un phénomène complexe. La connaissance de ses étapes successives serait essentielle pour en analyser ensuite les mécanismes.

Même si des facteurs du milieu extérieur peuvent moduler certaines étapes de l'argenture, celle-ci constitue, comme les autres métamorphoses, un évènement programmé du développement mettant en jeu une horloge interne. A ce sujet notons que la teneur en lipides du sang et des muscles pourrait constituer un signal important (LARSSON et al., 1990). Nous nous limiterons au rôle que peut jouer le système neuroendocrinien dans le déterminisme des phases successives de l'argenture.

\section{Les étapes}

On a mentionné plus haut les travaux de PANKHURST et de ses collaborateurs qui ont étudié l'intensité d'un certain nombre de changements liés à l'argenture en fonction du développement des follicules ovariens ; il en ressort que certains de ces changements débutent pour des diamètres folliculaires inférieurs à $0,1 \mathrm{~mm}$, c'est-à-dire la limite en dessous de laquelle on ne trouve pas d'animaux migrant. Des résultats de KLECKNER et KRUEGER (1981) vont dans le même sens. Ils suggèrent en effet (chez Anguilla rostrata) qu'au cours de l'année la longueur des capillaires du réseau admirable de la vessie natatoire commence à augmenter chez certains individus encore d'apparence jaune. Nous-mêmes, reconsidérant un ensemble de valeurs de RGS obtenues au cours de l'année chez des anguilles jaunes, avons observé en mai-juin une augmentation de la valeur moyenne et, surtout, du coefficient de variation de ce paramètre. On doit rappeler à ce sujet d'anciens travaux de RODOLICO (1933) qui avait observé, chez des mâles, une augmentation du tissu interstitiel du testicule avant le changement de pigmentation. Tout ceci suggère que l'argenture au sens large débute au printemps, largement avant les changements de coloration et de comportements dont on peut estimer qu'ils ne deviennent nets qu'en août-septembre. 
En 1993, nous avons étudié de ce point de vue deux ensembles d'anguilles femelles de poids moyens voisins de $230 \mathrm{~g}$. Les unes avaient été obtenues début juillet grâce à une opération de pêche électrique effectuée par les services du Conseil Supérieur de la Pêche dans la rivière Arques, à quelque $5 \mathrm{~km}$ au sud de Dieppe (Somme). Les autres - et c'est l'origine habituelle de nos animaux d'expérience - avaient été obtenues en octobre-novembre à la suite de pêches professionnelles effectuées lors de l'avalaison des poissons au débouché d'un étang situé près de Péronne (Somme). A priori, les premières étaient donc supposées jaunes et les secondes argentées, ce qui ne s'est révélé que grossièrement exact.

Nous nous sommes en particulier intéressés aux paramètres suivants : rapport gonadosomatique (RGS), index oculaire (IO), rapport du poids de l'intestin au poids du corps (RIS) et rapport du poids du foie au poids du corps (RHS) qui sont tous significativement différents entre les deux ensembles d'anguilles. Un certain nombre de dosages hormonaux, ainsi que celui des triglycérides dans plusieurs tissus, ont aussi été effectués. L'étude histologique des ovaires est en cours.

Les résultats obtenus seront exposés en détail ultérieurement (FONTAINE, HARDY, LE BELLE, MARCHELIDON, VIDAL et DUFOUR, en préparation), mais nous présenterons brièvement ici quelques données montrant que la situation est plus complexe qu'on pouvait le penser. Un histogramme de fréquence des RGS établi sur l'ensemble des animaux nous a montré qu'il existait non pas deux mais trois pics bien séparés. Ceci suggère qu'il existe deux étapes distinctes dans le développement de l'ovaire, avec un RGS passant respectivement de 0,2-0,4 à environ 0,9 puis de cette dernière valeur à environ 1,5. D'un intérêt particulier est le fait qu'au cours de la première étape (préargenture 1, tableau 1), il n'y a pas modification de paramètres qu'on peut qualifier de métamorphiques (IO, RIS) ni de RHS ; par contre une augmentation brutale des triglycérides (TG) du muscle blanc (de 1-4 à 20-75 mg/g) en marquerait le début. Au cours de la seconde étape (préargenture 2, tableau I), le rapport RGS/volume folliculaire augmente moins vite que durant la première, ce qui suggère que l'atrésie folliculaire se déroule alors. Quant aux changements métamorphiques, ils semblent encore limités avec seulement des indices d'augmentation de' 10 et aussi des indices de changement de coloration. Ces modifications métamorphiques prennent toute leur ampleur durant une troisième étape qui apparaît correspondre à la migration catadrome et que nous appellerons pour cette raison «avalaison" (tableau I). On peut supposer que la préargenture 2 inclut aussi l'apparition d'une excitation et d'une hyperactivité motrice, et des changements branchiaux qui conduisent ensuite au déséquilibre osmotique, à l'asthénie, et à l'avalaison. Des données supplémentaires seraient nécessaires pour donner une place, dans cette chronologie, à des évènements importants comme l'instauration du jeûne ou celle du blocage de la fonction gonadotrope qui a été mis en évidence chez l'anguille argentée (DUFOUR, 1985 et ce numéro ; DUFOUR et al., 1991 ; FONTAINE et DUFOUR, 1991).

II serait intéressant de disposer de caractères quantifiables et aisément mesurables permettant de juger du stade atteint par les anguilles et nos résultats préliminaires indiquent que 10 , l'index oculaire, peut être utile à cet égard. En effet chez les individus n'ayant pas entamé leur préargenture, il est égal à $4,33 \pm 0,24$ avec des limites (moyenne $\pm 2 s$ ) égales à 2,94 et 5,70 tandis que chez les individus ayant effectué les trois étapes de l'argenture il est de 8,48 $\pm 0,42$ avec des limites (moyenne $\pm 2 \mathrm{~s}$ ) égales à 5,58 et 11,4. Ces deux stades sont donc assez clairement distingués par 10 et ce paramètre pourrait être utile pour la gestion des stocks. On peut, par exemple, émettre l'hypothèse que, parmi une population d'anguilles jaunes, celles ayant un 10 supérieur à 5,6 (à titre indicatif, il s'agissait d'un tiers de l'échantillon très limité que nous avons étudié) et ayant donc sans doute amorcé leur argenture effectueront leur avalaison dans l'année. Toutefois une telle conclusion reste très approximative. En effet, nous avons observé parmi les anguilles d'avalaison des individus qui ne s'étaient pas vraiment métamorphosés, de "fausses argentées" en somme (avec par exemple un IO faible), et il est vraisemblable qu'il s'agit d'individus "à risque" quant à leur capacité de migrer jusqu'à la mer des Sargasses et donc de faire partie du stock de reproducteurs. De plus, on ne peut éliminer la possibilité que les diverses étapes de l'argenture s'échelonnent sur plus d'une année et que des anguilles jaunes subissent part de l'argenture sans pour autant effectuer l'avalaison immédiatement. 
Tableau I : Etapes de l'argenture des anguilles femelles du nord de la France : hypothèses de travail.

Le passage du stade A au stade B puis celui du stade B au stade C s'effectuent pendant le printemps et l'été ; l'avalaison a lieu pendant l'automne et l'hiver.

+ ou - : augmentation ou diminution significative $(p<0,05)$. Pour 10 , l'augmentation est significative entre les stades $B$ et $D$. NS : changement non significatif.

Table I : Steps of female eel silvering in the north of France: working hypotheses.

The transitions from stage $A$ to stage $B$ then from stage $B$ to stage $C$ occur during spring and summer; downstream migration (= avalaison) occurs during fall and winter.

+ or - : significant $(p<0.05)$ increase or decrease. 10 increase is significant between stages $B$ and $D$. NS : non significant change.

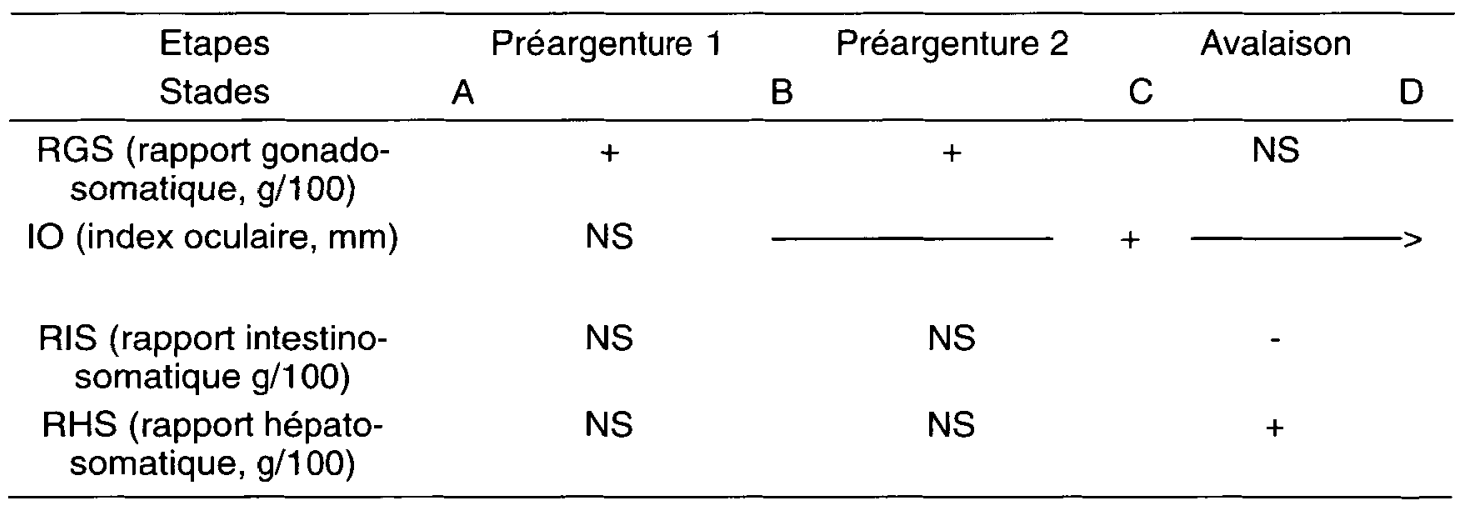

\section{Les mécanismes neuroendocriniens}

Ils sont en fait pratiquement inconnus mais je voudrais résumer ici un certain nombre de données qui peuvent orienter la recherche dans ce domaine. II s'agit des tentatives d'induction expérimentale de l'argenture, de la détermination de profils hormonaux au cours de celle-ci et des résultats obtenus sur la métamorphose d'autres espèces.

Deux observations faites chez des anguilles traitées au cortisol suggèrent un effet de cette hormone sur la pigmentation. Chez des mâles argentés, OLIVEREAU (1966) a noté que «les anguilles traitées apparaissaient très argentées et beaucoup plus pâles que les témoins". Chez des anguilles (Anguilla rostrata) d'eau douce pas autrement caractérisées, EPSTEIN et al. (1971) ont noté que «les animaux injectés avec du cortisol pendant plus de 7-10 jours perdaient leur couleur jaune et devenaient argentés". Nous-mêmes (FONTAINE et LE BELLE, données inédites) avons injecté des anguilles jaunes femelles (poids du corps voisin de $260 \mathrm{~g}$ ) pendant 3 semaines avec du cortisol ( $1 \mathrm{mg} 3$ fois par semaine). Une très légère tendance à un affaiblissement de la couleur jaune a été observée tandis qu'aucune différence n'a été observée entre les lots en ce qui concerne le RGS (voisin de 0,40 ) ou le diamètre oculaire. Un traitement similaire avec la triiodothyronine (T3; $2 \mu \mathrm{g} 3$ fois par semaine), hormone thyroïdienne généralement impliquée dans les métamorphoses, n'a eu aucun effet non plus que l'association cortisol-triodothyronine. Par contre, nous avons récemment observé chez des femelles traitées à la testostérone à la fois des changements de coloration et, surtout, une augmentation significative de l'index oculaire (DUFOUR, MONTERO, LE BELLE et FONTAINE, données inédites). Ce résultat suggère que la testostérone est impliquée dans les changements des pigments rétiniens observés par WOOD et PARTRIDGE (1993) chez des anguilles mâles sous l'effet de l'hormone gonadotrope chorionique humaine. 
Les données concernant les profils hormonaux durant l'argenture sont encore plus rares. LELOUP et al. (1976 et communication personnelle) ont observé une augmentation de la T3 circulante et du rapport T3 / thyroxine (T4) en fonction du RGS ; cette augmentation est déjà très nette (plus de 2 fois pour T3/T4) quand le RGS passe d'environ 0,2 à 0,7 c'està-dire bien avant qu'il atteigne les valeurs propres aux anguilles argentées. Ceci suggère une stimulation de la thyroïde dès l'étape, définie ci-dessus, de la préargenture 1 .

Nos connaissances sur d'autres métamorphoses peuvent aussi servir de supports à des hypothèses de travail. On connaît le rôle majeur des hormones thyroïdiennes dans la métamorphose des amphibiens. Au cours de cette dernière, des pics plasmatiques successifs de thyroxine, de cortisone et de prolactine (PRL) ont été mis en évidence chez Rana catesbeiana (DICKHOFF et al., 1990). Une augmentation, dans l'hypophyse, de la sous-unité $\alpha$ des hormones glycoprotéiques (TANAKA et al., 1991) et des ARN messagers codant pour l'hormone de croissance (TAKAHASHI et al., 1992) a également été démontrée. Chez les Téléostéens, la spectaculaire métamorphose larvaire des Pleuronectidés implique aussi les hormones thyroïdiennes et le cortisol (DE JESUS et al., 1990).

Un autre événement intéressant pour notre propos est la smoltification des Salmonidés. II s'agit, comme pour l'argenture, de l'expression d'adaptats anticipateurs qui prépare le poisson, en eau douce, à sa migration marine. Au cours de la transformation du parr en smolt, des pics plasmatiques successifs d'insuline, de prolactine, de T3 et de T4, d'hormone de croissance et de cortisol ont été mis en évidence (DICKHOFF et al., 1990 ; BOEUF, 1993). Des travaux effectués sur les Salmonidés, au cours de l'accommodation à l'eau de mer, nous renseignent de plus sur certains des effets de ces hormones. C'est ainsi que le cortisol, les hormones thyroïdiennes et la GH interviennent dans les changements branchiaux assurant l'hypoosmorégulation (références in FONTAINE, 1993 ; PRUNET et al., 1994). L'effet de la GH est au moins en partie dû à son effet sur la désiodation de T4 en T3 (LEBEL et LELOUP, 1992).

L'ensemble des données que nous venons de résumer suggère que de nombreuses hormones interviennent dans le déterminisme des phases successives de l'argenture de l'anguille : insuline, prolactine et, en tous cas, cortisol, hormones thyroïdiennes et GH.

De plus, étant donné le caractère original de cette métamorphose qu'est la stimulation rapide transitoire des gonades, on doit suspecter l'intervention de facteurs gonadotropes. On ne peut éliminer l'existence d'un pic bref d'hormone gonadotrope (GTH) de type II (GTHII) qui poserait une question intéressante. En effet, on sait que chez les anguilles argentées pêchées en avalaison, la fonction gonadotrope (production de GTH II) est au repos à cause d'un double blocage central (manque de sécrétion de gonadolibérine - $\mathrm{GnRH}-$, inhibition dopaminergique) (DUFOUR, 1985 ; DUFOUR et al., 1991) ; on devrait donc se demander quand s'instaure ce blocage et s'il existe déjà au stade jaune. Par ailleurs, un effet gonadotrope d'autres hormones ne peut être écarté : GTH de type I (bien que celle-ci n'ait pu jusqu'ici être mise en évidence chez l'anguille), hormone de croissance (GH), insuline ; cette hypothèse est renforcée par le fait que l'administration aux anguilles jaunes d'un extrait hypophysaire riche en GTH II ne copie pas toutes les modifications liées à l'argenture : le diamètre folliculaire n'augmente que faiblement et on n'observe pas d'atrésie folliculaire (LOPEZ et FONTAINE, 1990). De plus, l'intervention de la GH dans le contrôle de la vitellogenèse a été mise en évidence chez l'anguille (BURZAWA-GERARD et DELEVALLEE-FORTIER, 1992 ; BURZAWA-GERARD et al., ce numéro).

Il semble raisonnable de penser que des changements au niveau de facteurs hypothalamiques hypophysiotropes sont parmi les événements précoces de l'argenture, changements qui induiraient une sécrétion accrue de $\mathrm{GH}$ ainsi que d'hormones thyréotrope (TSH) et corticotrope (ACTH) et peut-être de GTH. De ce point de vue, il faut mentionner des résultats qui indiquent que la spécificité de peptides hypophysiotropes est différente chez les Vertébrés inférieurs de ce qu'elle est chez les Mammifères. Rappelons que, chez ces derniers, ont été en particulier caractérisés des facteurs de décharge distincts pour la TSH (thyrolibérine ou TRH), l'ACTH (corticolibérine ou CRH) et les GTH $(\mathrm{GnRH})$. Or, chez le poisson rouge, Carassius auratus, PETER et al., (1987) ont montré que la GnRH était capable de stimuler non seulement la décharge de GTH mais aussi celle de GH. Quant à la sécrétion de TSH, elle ne semble pas être, chez les Téléostéens, stimulée par la TRH. II semble en être de même chez les têtards de l'amphibien Rana catesbeiana où, par contre, 
la CRH exerce cet effet (DENVER et LICHT, 1989) ; chez des amphibiens adultes, la TRH, la $\mathrm{CRH}$ et aussi la GnRH apparaissent capables de stimuler l'axe hypophysothyroïdien (JACOBS et al., 1988).

Si des situations similaires existent chez l'anguille, la stimulation concertée de la sécrétion des hormones hypophysaires apparemment importantes au cours de l'argenture pourrait être le fait d'un petit nombre de types neuronaux. C'est ainsi que l'activation de neurones à $\mathrm{CRH}$ et à $\mathrm{GnRH}$ pourrait entraîner respectivement, d'une part, la sécrétion de TSH et d'ACTH avec augmentation de la T4 et du cortisol et, d'autre part, la sécrétion de GTH et de GH.

En ce qui concerne la $\mathrm{CRH}$, il est intéressant de noter que ce neuropeptide, impliqué chez les Mammifères dans l'inhibition de l'appétit (MORLEY, 1987), est susceptible d'intervenir dans l'installation du jeûne synchonique.

La stimulation de la sécrétion ou de l'action de certains neuropeptides pourrait aussi passer par la levée des inhibitions exercées par d'autres facteurs hypothalamiques comme la dopamine (pour l'axe GnRH-GTH) ou la somatostatine (pour la GH et la TSH) (LIN et al., 1993).

\section{UN SCÉNARIO ÉVOLUTIF}

Le genre Anguilla fait partie des élopomorphes dont tous les représentants actuels ont une larve leptocéphale et qui sont apparus il y a environ 150 millions d'années (LAUDER et LIEM, 1983). Etant donné que tous les élopomorphes autres que les anguilles (par exemple, les congres) sont exclusivement marins, ces dernières doivent elles-mêmes descendre d'ancêtres qui l'étaient aussi. Quelles hypothèses peut-on formuler sur le scénario évolutif qui a conduit de cette situation au cycle vital caractéristique des anguilles actuelles?

Nous supposons d'abord que, au cours de l'évolution, un élément de ce cycle est resté constant, à savoir la reproduction dans des zones marines profondes bien définies, à cause d'une nécessité biologique dont nous ignorons les raisons (DUFOUR, 1986 ; FONTAINE, 1989). Notons que les congres, au cours de leur maturation sexuelle, migrent eux-mêmes en profondeur (HOOD et al., 1988). Ensuite, une étape importante de l'évolution des anguilles aurait été la conquête progressive, après métamorphose larvaire, des eaux littorales, des estuaires puis des eaux douces susceptibles d'avoir été plus riches en ressources alimentaires et plus pauvres en prédateurs que l'océan. Une telle conquête nécessitait l'acquisition de l'euryhalinité, c'est-à-dire des capacités de s'accomoder aussi bien à un environnement hypoosmotique qu'à un environnement hyperosmotique. Un adaptat essentiel a sans doute été la capacité de différencier et de stimuler des types variés de cellules à chlorure branchiales. Les poissons sténohalins marins possèdent des cellules $\alpha$ et des cellules "accessoires" tandis que les poissons sténohalins dulcicoles possèdent des cellules $\alpha$ et $B$ (PISAM et al., 1990). Quant aux euryhalins, ils possèdent déjà en eau douce des cellules $\alpha$ et des cellules accessoires, ces deux types cellulaires se modifiant en eau de mer. De plus, des cellules B ont été ou non - selon l'espèce étudiée - identifiées en eau douce ; lorsqu'elles y sont présentes, elles disparaissent en eau de mer (PISAM et RAMBOURG, 1991 ; PISAM, communication personnelle). Quoiqu'il en soit, les poissons euryhalins sont capables de modifier leur équipement branchial en cellules à chlorure en réponse à un nouvel environnement.

L'acte suivant du scénario aurait été la "fixation» de la phase de croissance en eau douce par l'introduction dans le cycle vital du stade dit jaune, grâce à certains aspects de la métamorphose larvaire dont on peut imaginer qu'elle confère aux civelles des caractères de poisson dulcicole. L'argenture ultérieure, la seconde métamorphose, doit alors être vue comme la fermeture d'une parenthèse qui, en même temps, initie la migration nécessaire à la reproduction et prépare l'animal aux conditions qu'il rencontrera durant son voyage vers l'aire de ponte.

Les changements qui font ressembler l'anguille ayant subi l'argenture à un poisson marin ne se font plus à l'entrée en eau de mer comme chez la plupart des poissons euryhalins mais se produisent auparavant, en eau douce, et ils apparaissent donc comme 
anticipateurs (M. FONTAINE, 1983). Ainsi, tout se passe comme si, au cours de l'évolution, un processus d'accomodation (c'est-à-dire déclenché par un changement de milieu extérieur) était devenu un processus programmé du développement. Un scénario analogue a été proposé pour l'apparition de caractères adaptatifs très divers par exemple l'épaississement, déjà présent chez l'embryon, de la peau de la plante du pied humain. Le processus impliqué par de tels scénarios a été appelé "genetic assimilation"par WADDINGTON $(1957,1975)$; HO et SAUNDERS (1979) ont suggéré que "some random mutation (may) occur in the genome of the organisms that would take over the role of the environment". L'argenture de l'anguille est un modèle intéressant à cet égard car on peut formuler des hypothèses de travail susceptibles de rendre compte d'une assimilation génétique. Supposons une situation ancestrale où existaient, d'une part, la suite programmée d'évènements conduisant à la puberté et, d'autre part, des capacités d'accommodations diverses à l'eau de mer profonde (par exemple des capacités de modifier certaines cellules branchiales). Pour passer à la situation actuelle, il suffit qu'une hormone A impliquée dans la puberté devienne capable de contrôler un tissu intervenant dans l'accommodation, ce qui peut se réaliser simplement par un changement dans l'expression des récepteurs de A. L'horloge interne initiant la puberté prendrait ainsi le contrôle de caractères qui, d'accommodateurs, deviendraient programmés. Nous nous proposons de chercher à identifier les éléments neuroendocriniens susceptibles d'avoir été mis en jeu dans un tel scénario. L'existence de récepteurs de la testostérone dans les bâtonnets rétiniens pourraient, à la lumière des résultats rapportés ci-dessus, constituer l'un de ces éléments.

Une fois établi le schéma général de leur cycle biologique, les anguilles européennes ont dû en modifier les modalités étant donné la dérive des continents qui a éloigné leurs aires de croissance et de ponte (DUFOUR, 1986 ; FONTAINE, 1989). II est vraisemblable que les adaptations nécessaires se sont effectuées par addition de nouveaux caractères comme l'augmentation des réserves énergétiques. De plus en plus, dans ces conditions, un strict contrôle du déroulement de la maturation sexuelle était nécessaire pour assurer la reproduction dans la lointaine aire de ponte. L'existence de verrous centraux de la fonction gonadotrope, ne pouvant être levés que par un séjour en profondeur, répond sans doute à cette nécessité.

\section{CONCLUSIONS}

L'argenture de l'anguille est un phénomène complexe tant par la diversité des changements qui la constituent que par leur chronologie. Pour la femelle, que nous avons surtout considérée ici, nous suggérons que l'argenture débute par deux phases successives et distinctes de développement des gonades, la plupart des évènements de nature plus métamorphique ne commençant à se manifester qu'avec la seconde d'entre elles. Ceci renforcerait l'hypothèse que l'initiation de la métamorphose est dépendante chez l'anguille - par des mécanismes encore inconnus — de l'horloge interne déclenchant la puberté.

D'un point de vue fondamental, la nature de ce couplage entre puberté et métamorphose constitue une des questions les plus intéressantes posées par l'argenture de l'anguille. Les dosages hormonaux en cours, ainsi que ceux que nous nous proposons d'effectuer sur des animaux à des stades variés, nous permettront de préciser des hypothèses de travail qu'une expérimentation ultérieure devra permettre d'évaluer.

D'un point de vue plus appliqué, la reconnaissance des stades atteints par les différents individus d'une population peut être intéressante à plusieurs égards. C'est ainsi qu'on peut espérer évaluer la proportion d'une population sédentaire qui quittera dans l'année sa zone de croissance pour effectuer l'avalaison, une donnée utile pour la gestion des stocks. Nous avons souligné l'intérêt dans ce but de la mesure de l'index oculaire tout en marquant le caractère approximatif des enseignements qu'on peut tirer des données préliminaires actuellement disponibles.

L'argenture est sans aucun doute une phase critique du cycle vital de l'anguille. Sa description précise et l'élucidation de ses mécanismes constituent des conditions nécessaires pour la défense d'une espèce qui est maintenant en danger (BRUSLE, 1990). 


\section{REMERCIEMENTS}

Au Conseil Supérieur de la Pêche dont l'aide nous a permis de reprendre l'étude de l'argenture de l'anguille ; à sa Délégation régionale de Compiègne, en particulier MM. J.P. AUXIETRE, J.C. LUCCHETTA ainsi qu'à leurs collaborateurs, pour l'organisation et la réalisation de la pêche d'anguilles jaunes. A Gérard BENISTI pour sa participation à cette pêche. A Sylvie DUFOUR pour de fructueuses discussions sur le thème de cet article. A Jocelyne MARTIN et Nadine LE BELLE pour leur aide dans l'établissement de la bibliographie. A Maurice FONTAINE pour la photographie d'anguille jaune.

\section{BIBLIOGRAPHIE}

ANCONA U. d', 1960. The life-cycle of the atlantic eel. Symposia of the zoological Society of London, 1, 61-67.

BARNI S., BERNOCCHI G., GERZELI G, 1985. Morphohistochemical changes in hepatocytes during the life cycle of the european eel. Tissue and Cell., 17, 97-109.

BEATTY D.D., 1975. Visual pigments of the american eel Anguilla Rostrata. Vision. Res., 15, 771-776.

BERTIN L., 1951. Les anguilles. Payot, Paris.

BERTIN L., 1958. Larves et métamorphoses. In «Traité de Zoologie», XIII, 3, 1813-1834. P. P. Grassé ed., Masson, Paris.

BOEUF G., 1993. Salmonid smolting : a pre-adaptation to the oceanic environment. In Fish Ecophysiology, 105-135. J.C. Rankin, F.B. Jensen eds., Chapman et Hall, Londres.

BOETIUS I., BOETIUS J.,1985. Lipid and protein content in Anguilla anguilla during growth and starvation. Dana, 7, 1-17.

BOSTRÖM S. L., JOHANSSON R.G., 1972. Enzyme activity patterns in white and red muscle of the eel (Anguilla anguilla) at different developmental stages. Comp. Biochem. Physiol., 42 B, 553-542.

BRUSLE J., 1990. L'Anguille européenne, Anguilla anguilla, une espèce jugée commune jusqu'à la dernière décennie, mérite-t-elle d'acquérir aujourd'hui le statut d'espèce menacée ? Bull. Soc. Zool., 114, 61-73.

BURZAWA-GERARD E., DELEVALLEE-FORTIER B.,1992. Implication de l'hormone de croissance au cours de l'induction expérimentale de la vitellogenèse par l'oestradiol $17 B$ chez l'anguille argentée (Anguilla anguilla L.). C.R. Acad. Sci. Paris, 314, 411-416.

BURZAWA-GERARD E., BALOCHE S., LELOUP-HATEY J., LE MENN F., MESSAOURI H., NUNEZ-RODRIGUEZ J., PEYON P., ROGER C., 1994. Ovogenèse chez l'anguille, Anguilla anguilla : ultrastructure de l'ovaire à différents stades de développement et implication des lipoprotéines au cours de la vitellogenèse. Bull. Fr. Pêche Piscic., 335.

DAVE G., JOHANSSON M.J., LARSSON A., LEWANDER K., LIDMAN U., 1974. Metabolic and hematological studies on the yellow and silver phases of the european eel, Anguilla anguilla L.- II. Fatty acid composition. Comp. Biochem. Physiol., 47B, 583-591.

DE JESUS E.G., INUI Y., HIRANO T., 1990. Cortisol enhances the stimulating action of thyroid hormones on dorsal fin-ray resorption of flounder larvae in vitro. Gen. Comp. Endocrinol., 79, 167-173.

DENVER R.J., LICHT P., 1989. Neuropeptide stimulation of thyrotropin secretion in the larval bullfrog : evidence for a common neuroregulator of thyroid and interrenal activity in metamorphosis. The J. of Exper. Zool., 252, 101-104.

DICKHOFF W.W., BROWN C.L., SULLIVAN C.V., BERN H.A., 1990. Fish and Amphibian models for developmental endocrinology. The J. of Exper. Endoc., 4, 90-97. 
DUFOUR S., 1985. La fonction gonadotrope de l'anguille européenne, Anguilla anguilla, au stade argenté (au moment du départ pour la migration de reproduction) : les mécanismes de son blocage et sa stimulation expérimentale. Thèse Muséum national d'Histoire naturelle et Université Paris VI.

DUFOUR S., 1986. La Saga des anguilles. La Recherche, 17, 1456-1459.

DUFOUR S., 1994. Neuroendocrinologie de la reproduction de l'anguille : de la recherche fondamentale aux problèmes appliqués. Buill. Fr. Pêche Piscic., 335.

DUFOUR S., BASSOMPIERRE M., MONTERO M., LE BELLE N., BALOCHE S. et FONTAINE Y.A, 1991. Stimulation of pituitary gonadotropic function in female silver eel treated by a gonadoliberin agonist and dopamine antagonists. In «Proc. IV th International Symposium on Reproductive Physiology of Fish". AP. Scott, JP Sumper, D.E. Kime and M.S. Rolfe, eds., Fish Symp. 91, Publ. Sheffield, 54-56.

EGGINGTON S., 1986. Metamorphosis of the american eel, Anguilla rostrata Lesueur. I. Changes in metabolism of skeletal muscle. The J. Exper. Zool., 237, 173-184.

EPSTEIN F.H., CYNAMON M., Mc KAY W., 1971. Endocrine control of Na-K-ATPase and seawater adaptation in Anguilla rostrata. Gen. Comp. Endocrinol. 16, 323-328.

FONTAINE M., 1975. Physiological mechanisms in the migration of marine and amphihaline fish. Adv. Mar. Biol., 13, 241-355.

FONTAINE M., 1983. Introduction à une écophysiologie d'anticipation. Bull. Soc. Ecophysiologie, 8, 63-69.

FONTAINE M., 1985. Le roman des anguilles. Bull. Soc. Sc. Nat. Ouest France, 7, 27-40.

FONTAINE M. , OLIVEREAU M., 1962. Nutrition et sexualité chez les poissons. Ann. Nutr. Alim., 16, A125-A152.

FONTAINE Y.A., 1989. Les anguilles : migration et reproduction. Océanis, 15, 197-206

FONTAINE Y.A. 1993. Adaptations versus accomodations : some neuroendocrine aspects in teleost fish. Fish Physiol. Biochem., 11, 147-154.

FONTAINE Y.A., 1994. Adaptation et accomodation. Stratégies d'espèces et stratégies individuelles. In "Les Evolutions, phylogenèse de l'individuation", 129-142.Colloques de la Revue internationale de Psychologie. Presses universitaires de France. Paris.

FONTAINE Y.A., LOPEZ E., DELERUE-LE BELLE N., FONTAINE-BERTRAND E., LALLIER F., SALMON C., 1976. Stimulation gonadotrope de l'ovaire chez l'anguille (Anguilla anguilla L.) hypophysectomisée. Morphologie, activités adényl cyclase et phosphodiestérase de l'adénosine monophosphate cyclique. J. Physiol., Paris, 72, 871-892.

FONTAINE Y.A., DUFOUR S., FONTAINE M.,1985. L'immersion prolongée en profondeur stimule la fonction hypophysaire gonadotrope de l'anguille européenne (Anguilla anguilla L.) femelle. C. R. Acad. Sci. Paris, Serie III, 300, 83-87.

FONTAINE Y.A., DUFOUR S., 1991. The eels : from life cycle to reproductive endocrinology. Bull. Inst. Zool. Academica Sinica, Monograph 16, 237-248.

GOULD S., LEWONTIN R.C., 1979. The spandrels of San Marco and the panglossian paradigm : a critique of the adaptationnist programme. Proc. Roy. Soc. London, B 205, 581-598.

HO M. W., SAUNDERS P.T., 1979. Beyond neo Darwinism. An epigenetic approach to Evolution. J. Theor. Biol., 78, 573-591.

HOOD P.B., ABLE K.W., GRIMES C.B., 1988. Biology of the conger eel Conger oceanicus in the Mid-Atlantic Bight. I. Distribution, age, growth and reproduction. Marine Biol., 98, 587-596. 
JACOBS G.F.M., GOYVAERTS M.F., VANDORPE B., QUAGHEBEUR A.M.I., KUHN E.R., 1988. Luteinizing hormone releasing hormone as potent stimulator of the thyroidal axis in ranid frogs. Gen. Comp. Endocrinol., 70, 274-283.

KLECKNER R.C., 1980. Swimblader wall guanine enhancement related to migratory depth In silver phase Anguilla rostrata. Comp. Biochem. Physiol., 65 A, 351-354.

KLECKNER R.C., KRUEGER W.H., 1981. Changes in swimbladder retial morphology in Anguilla rostrata during premigration metamorphosis. J. Fish. Biol., 18, 569-577.

LARSEN L.O., DUFOUR S., 1993. Growth, reproduction and death in lampreys and eels. In "Fish Ecophysiology", 72-103, Rankin J.C. and Jensen F.B. ed, Chapman and Hall, London.

LARSSON P., HAMRIN S., OKLA L., 1990. Fat content as a factor inducing migratory behavior in the eel (Anguilla anguilla) to the Sargasso sea. Naturwissench. 77, 488-490.

LAUDER G.W., LIEM K.F., 1983. The evolution and interrelationships of the actinopterygian fishes. Bull. Mus. Comp. Zool., 150, 95-197.

LEBEL J.M. , LELOUP J., 1992. La triiodothyronine est nécessaire à l'acclimatation à l'eau de mer de la truite fario (Salmo trutta) ou arc-en-ciel (Oncorhynchus mykiss). $C$. $R$. Acad Sci., 314, 461-468.

LECOMTE-FINIGER R., 1990. Métamorphose de l'anguille jaune en anguille argentée (Anguilla anguilla L.) et sa migration catadrome. Ann. Biol., 29, 183-194.

LELOUP J., BRICHON G., HARDY A., 1976. Variations des hormones thyroïdiennes circulantes chez un Sélacien et des Téléostéens en fonction de l'évolution génitale et de la salinité du milieu. J. Physiol., Paris, 72, Abstract 48 A.

LELOUP-HATEY J., 1964. Fonctionnement de l'interrénal antérieur de deux Téléostéens : le saumon atlantique et l'anguille européenne. Ann. Inst. Océanog., 42, 221-338.

LELOUP-HATEY J., HARDY A., QUERAT B., 1989. Responses of gonado-somatic indice and plasma levels of sex steroids to gonadotropic stimulation in freshwater european eel at yellow and silver stages. Gen. Comp. Endocrinol., 74, 251.

LEWANDER K., DAVE G., JOHANSSON M. J., LARSSON A., LIDMAN U.,1974. Metabolic and hematological studies on the yellow and silver phases of the european eel, Anguilla anguilla L. I. Carbohydrate, lipid, protein and inorganic ion metabolism. Comp. Biochem. Physiol., 47B, 571-581.

LIN X.W., LIN H.R., PETER R.E., 1993. Growth hormone and gonadotropin secretion in the common carp (Cyprinus carpio L.) : in vitro interactions of gonadotropin-releasing hormone, somatostatin, and the dopamine agonist apomorphine. Gen. Comp. Endocrinol., 89, 62-71.

LOPEZ E., FONTAINE Y.A., 1990. Stimulation hormonale, in vivo, de l'ovaire d'anguille européenne au stade jaune. Reprod. Nut. Dév., 30, 577-582.

MORLEY J.E., 1987. Neuropeptide regulation of appetite and weight. Endoc. Rev. 8, 256-287.

OLIVEREAU M. 1966. Effet d'un traitement par le cortisol sur la structure histologique de l'interrénal et de quelques tissus de l'anguille. Ann. Endoc., 27, 549-560.

PANKHURST N.W., 1982 a. Relation of visual changes to the onset of sexual maturation in the European eel Anguilla anguilla L. J. Fish Biol., 21, 127-140.

PANKHURST N.W., 1982 b. Changes in body musculature with sexual maturation in the European eel, Anguilla anguilla L. J. Fish Biol., 21, 417-428.

PANKHURST N.W., LYTHGOE J.N. 1982. Structure and colour of the integument of the European eel Anguilla anguilla L. J. Fish Biol., 21, 279-296

PETER R.E., HABIBI H.R., MARCHANT T.A., NAHORNIAK C.S., 1987. Vertebrate gonadotropin-releasing hormones: phylogeny and structure-function relationship. Ann. N.Y. Acad. Sci., 519, 299-309. 
PISAM M., BOEUF G., PRUNET P., RAMBOURG G.A., 1990. Ultrastructural features of mitochondria rich cells in stenohaline freshwater and seawater fishes. The Amer. J. Anat., 187, 21-31

PISAM M., RAMBOURG A., 1991. Mitochondria rich cells in the gill epithelium of teleost fishes : an ultrastructural approach. Int. Rev. Cytol., 130, 191-231.

PRUNET P., PISAM M., CLAIREAUX J.P., BOEUF G., RAMBOURG A., 1994. Effects of growth hormone on gill chloride cells in juvenile Atlantic salmon. Am.J. Pysiol., 266 ; R850-R857.

RODOLICO A., 1933. Differenziamento dei sessi ed ovo-spermatogenesi nell'anguilla. Pub. St. Zool. Nap., Naples, 13, 180-278.

SORENSEN P.W., PANKHURST N.W., 1988. Histological changes in the gonad skin, intestine and olfactory epithelium of artificially-matured male american eels, Anguilla rostrata. J. Fish Biol. , 32, 297-307.

TAKAHASHI N., KIKUYAMA S., GEN K. MARUYAMA O., KATO Y., 1992. Cloning of a bullfrog growth hormone cDNA : expression of growth hormone mRNA in larval and adult bullfrog pituitaries. J. Mol. Endoc. 9, 165-173

TANAKA S., SAKAI M., PARK M.K., KUROSUMI., 1991. Differential appearance of the subunits of glycoprotein hormones ( $\mathrm{LH}, \mathrm{FSH}$, and TSH) in the pituitary of bullfrog (Rana catesbeiana) larvae during metamorphosis. Gen. Comp. Endocrinol., 84, 318-327.

TESCH F.W., 1979. The eel, biology and management of anguillid eels. Chapman and Hall, London.

THOMPSON A.J., SARGENT J.R., 1978. Changes in the levels of chloride cells and (Na-K) dependant ATPase in the gills of yellow and silver eels adapting to seawater. $J$. Exp. Zool. 200, 33-40.

WADDINGTON C.H., 1957. The strategy of genes. Allen and Unwin, London.

WADDINGTON C.H., 1975. The evolution of an evolutionist. Cornell University press, Ithaca, New York.

WALD G., 1981. Metamorphosis : an overview. In "Metamorphosis, a problem in developmental biology", 2 nd edition, Gilbert L.I. et Frieden E., Ed. Plenum Press, New York.

WHITE B.A., NICOLL C.S., 1981. Hormonal control of Amphibian metamorphosis. In "Metamorphosis, a problem in developmental biology", 363-396. Plenum Press, New York.

WOOD P., PARTRIDGE J.C.,1993. Opsin substitution induced in retinal rods of the eel (Anguilla anguilla L.) : a model for G-protein-linked receptors. Proc. R. Soc. Lond. B, 254, 227-232.

ZACCHEI A.M., TAVOLARO P., 1988. Lateral line system during the life cycle of Anguilla anguilla L. Boll. Zool., 3, 145-153. 